\title{
EXTENSIONS OF ERGODIC ACTIONS AND GENERALIZED DISCRETE SPECTRUM
}

\author{
BY ROBERT J. ZIMMER
}

Communicated by P. R. Halmos, February 4, 1975

1. Introduction. In this paper we announce results concerning extensions of ergodic actions of locally compact groups. Our results about extensions, together with related results and applications, enable us to obtain a measure theoretic analogue of Furstenberg's work in topological dynamics, to extend several well-known aspects of ergodic theory, and to present a unified view of these various phenomena. The author wishes to thank Professor G. W. Mackey for many helpful suggestions and conversations.

2. Generalization of the von Neumann-Halmos-Mackey theory. By an ergodic $G$-space, where $G$ is a locally compact second countable group, we mean a Lebesgue space $(X, \mu)$ together with a Borel action of $G$ on $X$, under which $\mu$ is invariant and ergodic. $(X, \mu)$ is called an extension of the ergodic $G$-space $(Y, \nu)$ if there is a Borel $G$-map $p: X \rightarrow Y$ with $p_{*}(\mu)=\nu$. By decomposing $\mu$ with respect to $\nu$ over the fibers of $p, L^{2}(X)$ becomes a Borel $G$-Hilbert bundle over $Y$ [8]. In the study of a single $G$-space $X$, an important role is played by the decomposition of the representation of $G$ on $L^{2}(X)$ defined by translation. For the study of extensions, the decomposition of $L^{2}(X)$ into $G$-invariant subbundles over $Y$ plays an analogous role. Theorems $\mathrm{A}, \mathrm{B}$, and $\mathrm{C}$ are the generalization to extensions of the classical "discrete spectrum" theory of von Neumann and Halmos [4], [9], as generalized, in part, by Mackey [5]. The classical results and Mackey's generalization are included in these theorems as the special case in which $Y$ is one point.

Definition. $X$ has relatively discrete spectrum over $Y$ if $L^{2}(X)$ is the direct sum of finite dimensional $G$-invariant subbundles over $Y . X$ has relatively elementary spectrum over $Y$ if each of these subbundles can be taken to be one dimensional.

Definition. If $Y$ is an ergodic $G$-space and $K$ a compact group, a Borel map $c: Y \times G \rightarrow K$ is called a homomorphism, or cocycle, if for each $g$, $h \in G, c(y, h g)=c(y, h) c(y h, g)$ for almost all $y \in Y$.

AMS (MOS) subject classifications (1970). Primary 22D40, 28A65, 54H20; Secondary 22D10, 22D 30, 47A35. 
Theorem A (STructure theorem). If $p: X \rightarrow Y$ is an extension with relatively discrete spectrum, then there is (i) a compact group $K$ and a closed subgroup $H \subset K$, and (ii) a Borel cocycle $c: Y \times G \rightarrow K$, such that up to equivalence modulo null sets, $X=Y \times K / H$, $p$ is projection onto $Y$, and the action of $G$ on $X$ is given by $(y,[k]) g=(y g,[k] c(y, g))$. If $X$ has relatively elementary spectrum, we can take $K$ abelian and $H=\{e\}$.

THEOREM B (UNIQUeNESS). Let $Y$ be an ergodic G-space and $S$ the group under tensor product of equivalence classes of 1-dimensional Borel $G$-Hilbert bundles on $Y$. If $X$ is an extension of $Y$ with relatively elementary spectrum, and $S_{X}$ is the set of equivalence classes of one dimensional G-bundles appearing in the decomposition of $L^{2}(X)$ over $Y$, then $S_{X}$ is a countable subgroup of $S$. Furthermore, if $Z$ is another extension of $Y$ with relatively elementary spectrum, then $X$ and $Z$ are equivalent extensions if and only if $S_{X}=S_{Z}$

Theorem C (Existence). Let $S$ be as in Theorem B, and $T \subset S a$ countable subgroup. Then there is an extension $X$ of $Y$ with relatively elementary spectrum such that $S_{X}=T$.

For extensions with relatively discrete but not relatively elementary spectrum, the natural analogue of Theorem B is false even for $Y=\{e\}$ [5]. Nevertheless, by considering the notion of a normal extension, so called because in Mackey's theory of virtual subgroups [6] it is the analogue of a normal subgroup, one obtains a class of extensions, containing those with relatively elementary spectrum, for which versions of Theorems B and C hold.

Definition. Let $p: X \rightarrow Y$ as above, and let $y \rightarrow H_{y}$ exhibit $L^{2}(X)$ as a $G$-Hilbert bundle over $Y$. Then $x \rightarrow H_{p(x)}$ is a $G$-Hilbert bundle over $X$. $X$ is called a normal extension of $Y$ if this $G$-bundle is equivalent to a trivial $G$-Hilbert bundle on $X$.

TheOREM $\mathrm{B}^{\prime}$. Let $X$ and $Z$ be normal extensions of $Y$ with relatively discrete spectrum. Then $X$ and $Z$ are equivalent extensions of $Y$ if and only if the G-bundles they define are equivalent. In this case, the subgroup $H$ in Theorem A can be taken to be the identity.

THEOREM $\mathrm{C}^{\prime}$. Let $S$ be a countable collection of equivalence classes of finite dimensional irreducible G-bundles on an ergodic G-space $Y$, such that $S$ is closed under complex conjugation and contains every irreducible component of the tensor product of two elements of $S$. Then there is a normal 
extension $X$ of $Y$ such that $L^{2}(X)$ is equivalent as a $G$-bundle to $\Sigma_{\sigma \in S}(\operatorname{dim} \sigma) \sigma$.

3. Generalized discrete spectrum. We shall call actions that can be built up from a point by taking extensions with relatively discrete spectrum and inverse limits, actions with generalized discrete spectrum. In [7], Parry described a class of $Z$-actions, those with a separating sieve, that is a measure theoretic analogue of the class of distal actions. One can easily extend his definition to actions of an arbitrary group. The following is an analogue of Furstenberg's structure theorem for minimal distal flows [2].

TheOREM D. A nonatomic ergodic G-space has a separating sieve if and only if it has generalized discrete spectrum.

The next result exhibits the connection with quasi-discrete spectrum.

THEOREM E. A totally ergodic transformation has quasi-discrete spectrum [1] if and only if it is equivalent to a totally ergodic affine transformation [3] (on a compact, connected, abelian group) with generalized discrete spectrum.

The proofs of Theorems D and E depend upon (among other things) the following two results, which are of independent interest.

Theorem F. $L^{2}(X)$ has no nontrivial finite dimensional $G$-invariant subbundles over $Y$ if and only if the fibered product $X \times_{Y} X$ is ergodic.

THEOREM G. Let $H_{0} \subset L^{2}(X)$ be the sum of the finite dimensional G-invariant subbundles over $Y$, and $H_{1} \subset H_{0}$ the sum of the 1-dimensional $G$-invariant subbundles. Then there are intermediate $G$-spaces $W, V$ with $X \rightarrow W \rightarrow V \rightarrow Y$ such that $L^{2}(V)=H_{1}, L^{2}(W)=H_{0}$.

Further discussion, as well as detailed proofs, of these and other related results will appear elsewhere.

\section{REFERENCES}

1. L. M. Abramov, Metric automorphisms with quasi-discrete spectrum, Izv. Akad. Nauk SSSR Ser. Mat. 26 (1962), 513-530; English transl., Amer. Math. Soc. Transl. (2) 39 (1964), 37-56. MR 26 \#606.

2. H. Furstenberg, The structure of distal flows, Amer. J. Math. 85 (1963), 477515. MR $28 \# 602$.

3. F. J. Hahn, On affine transformations of compact abelian groups, Amer. J. Math. 85 (1963), 428-446. MR 27 \#889.

4. P. Halmos and J. von Neumann, Operator methods in classical mechanics. II, Ann. of Math. (2) 43 (1942), 332-350. MR 4, 14.

5. G. W. Mackey, Ergodic transformation groups with a pure point spectrum, Illinois J. Math. 8 (1964), 593-600. MR 30 \#3176. 
6. G. W. Mackey, Ergodic theory and virtual groups, Math. Ann. 166 (1966), 187-207. MR 34 \#1444.

7. W. Parry, Zero entropy of distal and related transformations, Topological Dynamics (Sympos., Colorado State Univ., Ft. Collins, Colo., 1967), Benjamin, New York, 1968, pp. 383-389. MR 38 \#2760.

8. A. Ramsay, Virtual groups and group actions, Advances in Math. 6 (1971), 253-322. MR 43 \#7590.

9. J. von Neumann, Zur Operatorenmethode in der klassichen Mechanik, Ann. of Math. 33 (1932), 587-642.

DEPARTMENT OF MATHEMATICS, HARVARD UNIVERSITY, CAMBRIDGE, MASSACHUSETTS 02138

\section{CORRIGENDUM, VOLUME 80}

G. I. Lehrer, Weil representations and cusp forms on unitary groups, pp. 11371141 .

In the section (§5) on applications of Theorem $A$ of [1], the statement of Corollary 2, p. 1140 is stronger than what is yielded by the argument outlined in the succeeding discussion.

It should be replaced by

Corollary 2. For $n$ odd, we have $\Sigma_{u \in R} J_{n}(u)=0$ for $R$ the unipotent radical of any proper parabolic subgroup of $U_{n}$. 